\title{
ARTICLE
}

\section{Release of MCBEND 11}

\author{
Pat Cowan*, Geoff Dobson and Julie Martin
}

AMEC, Kimmeridge House, Dorset Green Technology Park, Winfrith Newburgh, DT2 8ZB, United Kingdom

\begin{abstract}
MCBEND 11 is the latest version of the general radiation transport Monte Carlo code from AMEC's ANSWERS Software Service. MCBEND is well-established in the UK shielding community for radiation shielding and dosimetry assessments. The geometry visualisation software Visual Workshop 2A provides new event location and contoured results display for MCBEND11. Many important developments have been made available to users in this latest release of MCBEND, some of which are described in this paper. The geometry has been enhanced to import CAD generated input models in a tetrahedral mesh representation and five complex Hole geometries have been added. MCBEND11 has a new continuous energy collision processing package with associated nuclear data libraries, known as BINGO, and now supports one step coupled calculations. Enhancements have been made to the variance reduction capabilities include multiple importance maps and the ability to define a series of First Flight interfaces. The scoring capabilities have been enhanced by the provision of the Unified Tally module which allows scoring to be carried out in scoring bodies, which may be subdivided, and are independent of the geometry model. The Unified Tally module includes the capability to score fluxes and responses by material. An updated Response Function library and provision of a detector covariance library, both based on IRDF2002 data, are included with MCBEND11. New Random Number features provide support for Grid Processing calculations.
\end{abstract}

Keywords: MCBEND; Monte Carlo; radiation transport code; shielding

\section{Introduction}

MCBEND is a well-established powerful Monte Carlo software tool for general radiation transport analysis for shielding and dosimetry applications. MCBEND has been developed by AMEC in conjunction with Sellafield Ltd and is licensed for use by AMEC's ANSWERS Software Service. The MCBEND package comprises not only the Monte Carlo code itself but also nuclear data libraries, user documentation, productivity tools of various kinds and user support services. Supporting geometry model visualisation and verification tools are also available.

MCBEND is being continually developed to meet the needs of its users. MCBEND 11 is the next version to be released in line with the ANSWERS vision 'to provide easy-to-use software that meets the current and emerging needs of the user community'. In the case of MCBEND this vision focuses on the key areas of accuracy, understanding of uncertainties, efficiency and user-friendliness. This paper details the developments incorportated into MCBEND 11, covering improvements to the underlying physics and data libraries, improvements in calculation efficiency and improvements to the usability of the code.

*Corresponding author. Email: pat.cowan@amec.com

\section{Developments in MCBEND 11}

\subsection{Geometry modelling}

The MCBEND geometry modelling and tracking package comprises two components: Fractal ${ }^{\text {a }}$ Geometry (FG) which uses conventional ray tracing through geometrical bodies and Hole Geometry which uses Woodcock $^{\mathrm{b}}$ tracking. The same geometry modelling package is also available in the ANSWERS criticality code MONK.

\subsubsection{Fractal geometry}

Fractal Geometry is a well-established system of solid geometry modelling in which the problem geometry is subdivided into zones - defined as the intersections and differences of mathematical bodies. MCBEND has a large selection of body shapes ranging from simple bodies such as cuboids, cylinders and spheres to more complex bodies such as prisms, ellipses and tori. The bodies are assembled into structures called parts, which are self contained with their own local co-ordinate system to simplify the model construction. Parts may be

\footnotetext{
a The name 'Fractal' refers to the hierarchical nature where each sub unit or 'Part' could be a model in its own right. ${ }^{\mathrm{b}}$ A method that does no boundary intersection calculations.
} 
included within other parts to any depth of nesting and a given part may be included more than once within the geometry. The ability to break down complex models into parts simplifies the preparation and checking of the input data.

\subsubsection{Hole Geometry}

The Woodcock tracking algorithm in MCBEND is implemented via the Hole ${ }^{\mathrm{c}}$ Geometry package, and brings significant additional modelling power to the user. Hole geometries can be used to model common replicating arrangements and simple intersecting configurations in a short-cut form. MCBEND 11 supports the use of named holes and named materials in holes.

The Hole Geometry package continues to grow as additional hole types are added in response to industrial needs. MCBEND 11 incorporates five new hole geometries:

(a) PIPE HOLE - The PIPES hole geometry allows the user to model arrangements of pipes, with input parameters defining the location of the pipes; the radii of the annuli in the pipe; the materials or subsidiary holes in each annulus; the pairs of straight pipes to be linked by curved or straight pipes; and an interstitial material that may contain a subsidiary hole.

(b) BENTPINS HOLE This hole allows the user to model a set of curved pins. The pins may be deformed, either by being kinked or by being gently curved using a circular or sine-wave profile.

(c) VOXEL HOLE - The VOXEL hole is used for the approximate representation of an object as an array of cubic voxels.

(d) TETMESH HOLE geometry enables CAD generated tetrahedral mesh geometries to be imported into MCBEND 11 and treated as a Hole geometry.

(e) RANDOM RODS HOLE - The RANDROD hole models a random distribution of rods in a cylindrical container. The rods are all of the same length, radius and composition. Shown in Figure 1.

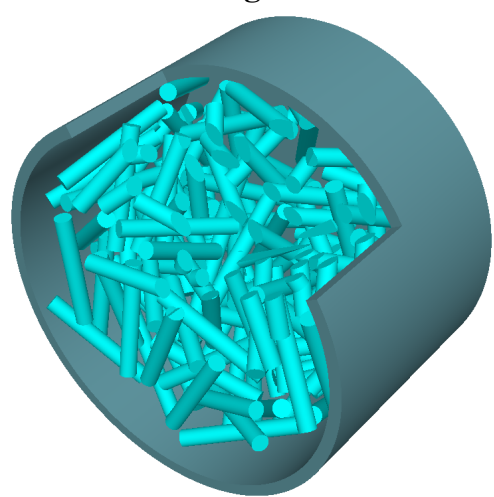

Figure 1. Random rods hole.

\subsubsection{Importing geometries created in CAD packages}

CAD geometries which have been converted into a tetrahedral mesh representation can then be imported

\footnotetext{
' A 'Hole' in MCBEND geometry context is a volume of space that uses Woodcock tracking.
}

into MCBEND as a TETMESH hole geometry.

A second method of importing CAD models allows an Initial Graphics Exchange Specification (IGES) model to be imported directly and used in combination with the existing geometry capabilities of MCBEND. All the surfaces in the CAD model are used directly with no approximation. This capability is provided by the OiNC software package developed by Sellafield Ltd. This method is a development feature in MCBEND 11 for evaluation purposes only.

Further details of both methods are given in a companion paper at this conference ${ }^{2}$.

\subsection{RCARD package enhancements}

The RCARD system of data input provides a powerful method of using mathematical and logical functions to construct a flexible and efficient input model.

MCBEND 11 has enhanced RCARD facilities including:

- Double precision arithmetic

- Exponentation to any power

- Functions INT, ATAN, ACOS and ASIN

- Variables containing character string values

- Variables containing logical conditions

- Parameters used for looping containing variables

- Nested IF statement blocks

- ELSEIF statements

- Loop counters in conditions

- Extended condition statements

- Additional RCARD controls

\subsection{Physics Features}

\subsubsection{BINGO collision processor}

Current and earlier versions of MCBEND use a module named DICE for neutron collision processing, together with DICE format nuclear data libraries. These libraries contain data derived from various evaluated files and the neutron cross-sections are stored on a fixed hyperfine energy grid.

MCBEND 11 contains a new collision processing package named BINGO, together with associated nuclear data libraries. BINGO libraries issued with MCBEND 11 include JEF2.2, JEFF 3.1 and ENDF/B VII. Data are available for neutrons, photons and electrons. Improvements in BINGO include: use of cross sections tabulated at energy points that are specific to each nuclide; improved variable temperature treatment; enhanced thermal scattering modelling including use of bound data for graphite; better representation of correlated energy/angle laws; more detailed representation of the tails of the fission spectrum; and explicit modelling of bremsstrahlung.

These capabilities give MCBEND 11 the tools to model complex systems with a greater degree of realism than with the DICE package.

\subsubsection{Coupled calculations}

Earlier versions of MCBEND execute a coupled 
neutron gamma calculation in two steps. In the first step a neutron calculation writes a collision file that contains all the events that could produce secondary gamma radiation. In the second step, the collision file is read and processed to derive sources for the gamma calculation. This method has been enhanced as it is now possible for the secondary gamma calculations to be executed by processing up to nine collision files which could be generated in parallel calculations.

MCBEND 11 also enables coupled neutron-gamma calculations to be executed in a single step for increased ease of use. MCBEND 11 also enables the summing of the dose from a neutron response and the dose from a gamma response to give a single result. A biological dose is an example of where this would be valuable.

\subsection{Variance reduction}

\subsubsection{Multi-map}

An importance map is used in MCBEND to control the splitting/Russian roulette $(\mathrm{S} / \mathrm{R})$ variance reduction process. It is defined in an orthogonal $\mathrm{XYZ}$ or R $\theta \mathrm{Z}$ mesh that is superimposed on the FG model.

The MULTIMAP feature allows different importance maps to be used in different parts of the FG model.

For the configuration shown in Figure 2 the objective of a MCBEND calculation is to track particles efficiently from inside the drums of active waste, out through the shielding of the drums, across the store and through the walls to the detector region of interest.

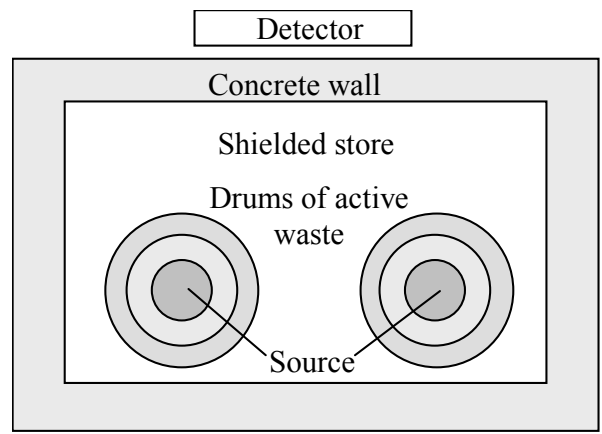

Figure 2. A simple shielding problem.

Neither of the previously available importance mesh systems are suitable for the whole geometry: an R $\theta Z$ mesh more comfortably fits the drums; an XYZ mesh more comfortably fits the store and its boundary walls.

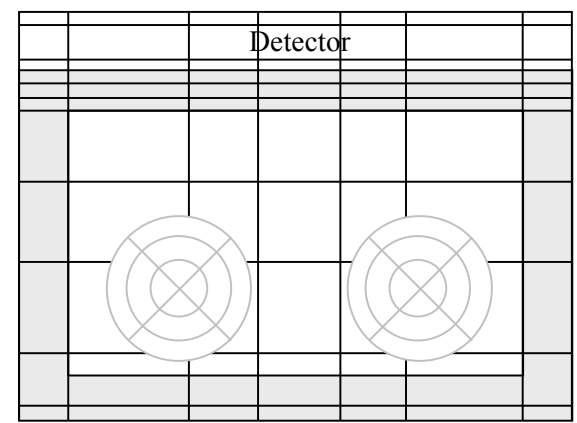

Figure 3. MULTMAP mesh example.

The MULTIMAP feature allows multiple importance maps to be specified in a MCBEND calculation. The MULTIMAP feature therefore allows the R $\theta Z$ mesh (light grey in Figure 3) in the source drums to be defined independently of the XYZ mesh over the remainder of the problem.

\subsubsection{Forced flight enhancements}

Forced flight variance reduction causes deterministic flights to be made from each source or collision point to user-defined surfaces. The method is typically used to direct particles into the mouth of a duct or collimator. A number of forced flight interface surfaces can be defined.

The forced flight module has been enhanced to allow a sequence of forced flight interfaces to be defined such that collisions after crossing one interface will lead to forced flights to the next in the sequence.

\subsection{Scoring}

\subsubsection{Unified tally module}

A new Unified Tally Module (UT) allows scoring to be carried out in sets of meshes that are independent of the geometry model and importance mesh. The method is based on the principle of scoring bodies or surfaces that may be subdivided to form localised scoring meshes of any required size. Any number of bodies may be defined and may overlap freely.

Each scoring body has a named shape e.g. BOX, ZROD and is located by its origin. The size of each scoring body is determined by shape parameters e.g. length, radius, height, and the bodies can be rotated as required. Each scoring body may be subdivided to allow localised scoring. All scoring bodies and their permitted methods of subdivision allow the code to calculate the volumes of the scoring mesh cells.

As many scoring bodies as required may be defined. The scoring in each body is independent of other bodies so that bodies may overlap. For example, it would be acceptable to superimpose two bodies of identical shape. One could use a fine internal mesh to obtain good resolution of results; the other could use a coarser mesh to obtain better statistics. Alternatively, superimposed bodies could be used for scoring in different group schemes. UT supports all MCBEND scores apart from sensitivities and energy deposition, and it may be used in conjunction with all existing scoring facilities.

\subsubsection{Scoring by material}

Scoring capabilities in the UT module also include scoring by material. This divides the scores recorded in a given mesh between the events in individual materials. It is principally intended for scoring results in the components of 'hole' geometries - as hole geometries do not have distinct 'zones' of different materials. Since tracking through such materials does not identify surface crossings it is not possible to use track length estimation; material scoring is therefore confined to collision density estimation. Essentially the material identified at a collision point becomes an additional subscript in the 
scoring registers. Normalisation of the results requires the volume of each material in each mesh. An option is therefore provided in the code to estimate relevant volumes by Monte Carlo.

\subsubsection{Response Functions}

The response function library used by MCBEND 11 allows the user access to a wide range of response functions in a detailed group scheme.

The new response library has been updated to include response functions from the IRDF-2002 dosimetry file, released in 2006. The IRDF2002 data have been processed using NJOY to produce a detector cross-section library and a detector covariance library for use with MCBEND 11. The cross-sections were processed into an ultrafine 13,230 energy group scheme that is fine enough to account for resonance self shielding of single resonance reactions and also gives a good representation of threshold reactions whilst the covariances were processed into 25 broad groups.

The ICRP-74 neutron and gamma-ray dose rate conversion factors are available. MCBEND 11 can calculate dose rates due to beta radiation

\subsection{Support for GRID calculations}

\subsubsection{Random number generator}

In GRID processing the same calculation is run on many processors, with independent random number sequences ${ }^{\mathrm{d}}$. This is achieved by advancing ('nudging') the random number generator by a different (and large) number of steps on each processor.

In MCBEND 11 this is achieved by using the same seeds, but nudging the random generator on by $n * 2^{39}$ where $\mathrm{n}$ is the processor number. Since only one of the generators used by MCBEND is nudged the sequence is independent on each processor even if more than $2^{39}$ numbers are used.

The results are then combined within MCBEND 11 enabling a complete GRID calculation to be easily achieved. Using the same random number sequence in a single calculation allows the user to exactly reproduce the results of a GRID calculation in a single run, providing a verification route for the GRID option.

\subsubsection{Merge multiple dump files}

A MCBEND dump file contains a snapshot of the state of a given calculation when the dump is written. It contains the values of all the numbers required for restarting the calculation from that point.

\footnotetext{
${ }^{d}$ MCBEND uses a combined Lagged Fibonacce and Linear Congrential generator. This gives a period of $2^{78}$ numbers.
}

MCBEND 11 is able to merge dump files in a restart case. The combination of dump files may then be used to print combined results, or to continue the calculation.

\subsection{Visual workshop}

Visual Workshop 2A has been developed to support ANSWERS Software Service codes. Visual Workshop $2 \mathrm{~A}$ provides $3 \mathrm{D}$ traced geometry display, geometry error checking, event location display and contoured results displays. Further details are given in a companion paper at this conference ${ }^{3}$.

\section{Conclusion}

This paper has summarised the developments incorporated into MCBEND 11. These cover improvements to the underlying physics and data libraries, improvements in calculation efficiency and improvements to the usability of the code. MCBEND continues to be focused on meeting the current and future needs of its customers.

\section{Acknowledgements}

The authors wish to acknowledge the other members of the MCBEND development team: A. Bird, E. Shuttleworth, M. Armishaw and G. Wright at AMEC, UK and A. Cooper at Sellafield Ltd.

\section{References}

[1] M. Armishaw and A.J. Cooper, Current Status and Future Direction of the MONK Software Package, Proc 8th International Conference on Nuclear Criticality Safety (ICNC 2007), St. Petersburg, Russia, May, 2007, (2007).

[2] A. Bird and A. Kyrieleis, Experience Using Models Imported From CAD Software For Shielding Calculations in MCBEND, Proc. 12th International Conference on Radiation Shielding (ICRS-12) and $17^{\text {th }}$ Topical Meeting on Radiation Protection and Shielding (RPSD-2012), Nara, Japan, September, 2012, (2012).

[3] A. Bird and T. Fry, Visual Workshop 2: A Model Viewer, Editor and Results Display Package for the Answers Shielding and Criticality Codes, Proc. 12th International Conference on Radiation Shielding (ICRS-12) and $17^{\text {th }}$ Topical Meeting on Radiation Protection and Shielding (RPSD-2012), Nara, Japan, September, 2012, (2012). 